\title{
Acromegaly and pregnancy: a prospective study
}

\author{
Monike Dias, Cesar Boguszewski ${ }^{1}$, Monica Gadelha ${ }^{2}$, Leandro Kasuki², \\ Nina Musolino ${ }^{3}$, Jose G H Vieira and Julio Abucham
}

Neuroendocrinology Unit, Division of Endocrinology, UNIFESP, Escola Paulista de Medicina da Universidade Federal de São Paulo, Rua Pedro de Toledo 910, 04039-002 São Paulo, Brazil, 'SEMPR - Endocrinology and Metabology Department, Universidade Federal do Paraná, Curitiba, Brazil, ${ }^{2}$ Neuroendocrinology Unit, Universidade Federal do Rio de Janeiro, Rio de Janeiro, Brazil and ${ }^{3}$ Neurosurgery Department, Universidade de São Paulo, São Paulo, Brazil

Correspondence

should be addressed to

J Abucham

Email

julioabucham@uol.com.br

\begin{abstract}
Context and objective: The interaction between pregnancy and acromegaly has been studied only retrospectively.

We used prospective data to assess those interactions.

Design: Prospective, interventional, multicentric study.

Patients: Ten pregnancies in eight acromegalic patients were included according to the following criteria: previous diagnosis of acromegaly; and active acromegaly before pregnancy. Sellar magnetic resonance image (MRI), GH, and IGF1

measurements were carried out before pregnancy. The exclusion criterion was radiotherapy.

Intervention: Withdrawal of pharmacological treatment (octreotide and/or cabergoline and/or pegvisomant) following pregnancy diagnosis.

Main outcome measures: Clinical/biochemical evaluations throughout pregnancy/puerperium and sellar MRI after delivery; and GH and IGF1 measurements before pregnancy. GH was measured by an interference-free IFMA assay during pregnancy and IGF1 by measured by Immulite 2000 assay in patients and 64 control pregnancies.

Results: No tumor growth was observed. Nine deliveries were at term and one at 35 weeks (preeclampsia). All newborns were healthy. Mean IGF1 levels before and during pregnancy were similar, but increased significantly during puerperium. As IGF1 in controls increased after midgestation, the prevalence of controlled IGF1 rose significantly from 2/10 ( $<20$ weeks) to 9/10 ( $>30$ weeks). Diabetes mellitus and hypertension/preeclampsia developed in one patient in each group; both complications were nonsignificantly $(P=0.06)$ associated with IGF1 $>1.3$ ULN before pregnancy.

Conclusions: Acromegaly control usually improved and tumor growth was not stimulated during pregnancy in spite of withdrawal of drug treatment. Drug treatment can be discontinued in most patients. Uncontrolled disease before pregnancy may pose a higher risk for diabetes and hypertension.
\end{abstract}

\section{Introduction}

Acromegaly is usually caused by a growth hormone (GH)secreting pituitary macroadenoma. Although fertility is frequently impaired (1), pregnancy is apparently becoming more common due to improvement in acromegaly treatment as well as in fertility therapies. As both acromegaly and pregnancy are associated with hypertension and diabetes, in uncontrolled acromegalic patients, pregnancy is reported to increase the prevalence of those comorbidities (2) and potentially complicate obstetrical/ fetal outcomes. In analogy to the effects of pregnancy on both tumoral (3) and nontumoral (4) lactotrophic cells, tumor enlargement and/or lactotrophic hyperplasia in acromegaly could also compress the optic chiasm and lead to visual impairment during pregnancy (5).

Clinical activity of acromegaly has been variably shown to improve $(6,7)$, remain stable $(8)$, or worsen during pregnancy (9), but no clear correlation with hormone levels has been established. Hormonal assessment of acromegaly during pregnancy is challenging. Interference of circulating placental hormones with (c) 2014 European Society of Endocrinology Printed in Great Britain
Published by Bioscientifica Ltd. 
homology to pituitary GH can often lead to either falsely elevated (10) or suppressed GH values in GH assays (11). Also, usual reference ranges for both basal and post glucose $\mathrm{GH}$ as well as for insulin-like growth factor 1 (IGF1) levels cannot be applied to pregnant women, as GH levels decline and IGF1 levels increase during normal pregnancy (12).

Optimal management of acromegaly during pregnancy has not been established. With the widespread use of pharmacological treatment to control disease activity, a consensus on acromegaly management has recently stated the need to encourage reporting of outcomes in medically treated pregnant patients (13). During pregnancy, pharmacological treatment with somatostatin analogs has been associated with decreased length in newborns (14), whereas cabergoline has been considered probably safe in prolactinomas (15), and pegvisomant, a GH receptor antagonist, has been reportedly used in only two cases $(16,17)$. Although cessation of medical therapy during pregnancy has been usually advised (13), this recommendation results primarily from the lack of a large database on drug safety and not from prospective studies on pregnancy outcomes following drug withdrawal.

In an extensive search of the literature, no prospective studies on pregnancy and acromegaly were found. Therefore, we conducted a prospective study to analyze the clinical, hormonal, tumoral, and maternal-fetal outcomes in acromegalic pregnant patients following withdrawal of pharmacological therapy.

\section{Subjects and methods}

\section{Study protocol}

The study was approved by our Institution's ethical committee and all participating patients and controls signed an informed consent. Inclusion criteria were: i) pregnancy with previous diagnosis of acromegaly; ii) high level of IGF1 before medical treatment and/or after pituitary surgery; iii) available sellar magnetic resonance image (MRI); and GH and IGF1 measurements before pregnancy. Exclusion criterion was patients who had undergone radiotherapy previously. Pharmacological treatment was withdrawn as soon as pregnancy was diagnosed (5-6 weeks). Patients were clinically and biochemically evaluated throughout pregnancy with at least one visit per trimester and at least one visit after delivery. In all ten patients, blood was collected for hormonal evaluation at least once in each trimester of pregnancy, between weeks 4 and 39, not always in the same gestational week, except for patient $\mathrm{G}$ who missed her second trimester hormonal assessment. Screening for diabetes mellitus was performed between 24 and 28 weeks. In each visit, symptoms were registered, physical and visual field examinations were assessed, and blood was collected for hormonal measurements. Sellar MRI was repeated between 1 and 24 weeks after delivery, usually during lactation. Lactation was allowed and the first clinical and hormonal evaluation during puerperium was performed between 3 and 6 weeks after delivery, usually during lactation. The decision to resume drug treatment and stop lactation was individualized according to clinical judgment.

Blood was collected from 64 women with normal singleton pregnancies at different gestational ages between weeks 5 and 39 and used as controls for GH and IGF1 values.

\section{Hormonal measurements}

All patients and controls had blood samples collected in the morning, after an overnight fast. Samples for measuring GH and IGF1 levels were usually collected at a single time point. Patients who had two or three blood collections for measurement of GH at 15-30 min intervals had their GH levels expressed by the mean value. Serum was separated and kept at $-20^{\circ} \mathrm{C}$ for $1-12$ weeks before the assays.

IGF1 assay - IGF1 was measured by an immunometric chemiluminescence assay (Immulite 2000, Siemens Medical Solutions Diagnostics, Los Angeles, CA, USA). Intra- and interassay coefficients of variation $(\mathrm{CV})$ are $<3.9$ and $<8.1 \%$ respectively. IGF1 levels were reported in relation to the upper limit of normal for age in a Brazilian population (18).

GH assays during pregnancy $\downarrow$ Initially (pregnancies A1-D5), GH was only measured by a GH assay, which showed an unusual negative interference, as we reported elsewhere $(11,19)$. Therefore, valid GH measurements could not be obtained in those five patients during pregnancy. In the last pregnancies (D6-H10) and in controls, GH was measured using a recently developed interference-free immunofluorometric $\mathrm{GH}$ assay validated to measure serum GH level during pregnancy and pegvisomant treatment (11). Intra-assay $\mathrm{CV}$ were $<2.9 \%$ and interassay $\mathrm{CV}$ were $<7.5 \%$. Crossreactivity studies with two commercially available nonglycosylated human placental GH (pGH) isoforms were $<0.001 \%$ for both $20 \mathrm{~K}$ and $22 \mathrm{~K}$ pGH in that assay (11). 
GH assays before and after pregnancy - Before and after each pregnancy, GH levels were measured by different routine assays in the various referring centers. Thus, we could not compare GH levels before or after pregnancy with those obtained by our interference-free assay during pregnancy. Likewise, no comparisons were made between $\mathrm{GH}$ concentrations obtained by different assays. However, as each patient had both pre- and post-pregnancy $\mathrm{GH}$ levels determined by the same assay, the percent differences between those measurements were calculated.

\section{Statistical analysis}

Statistical analyses were performed using GraphPad software program (version 5.0, GraphPad Software, Inc., San Diego, CA, USA 2007). Gestational age was defined as weeks after the last menstrual period. Individual values of GH or IGF1 concentrations represent the mean of two to three different measurements obtained along that period of time in most patients. Individual IGF1 measurements were expressed in relation to the upper limit of normal values for age according to the formula: patients' IGF1 concentration divided by the upper limit of normality of IGF1 for age in a Brazilian population (18). Percent changes in hormone levels between two conditions in the same patient were calculated by the formula: ((hormone concentration in the later conditionhormone concentration in the former condition)/ concentration in the former condition) $\times 100$.

Individual GH and IGF1 levels during pregnancy in patients were grouped according to gestational age based on changes in IGF1 levels observed along normal pregnancy in controls. Parametric (paired or unpaired t-tests, or ANOVA followed by Bonferroni's multiple comparison test or $P$ for trend) and nonparametric tests (Wilcoxon matched pairs test, Mann-Whitney $U$ test, or Kruskal-Wallis test followed by Dunn's multiple comparison test) were used according to data distribution. Fisher's exact test was used to analyze two categorical variables. The $\chi^{2}$ test for trend was used to define tendency of three frequencies along time. Significance was set at $P<0.05$.

\section{Results}

Fifteen pregnancies in 13 acromegalic patients were initially enrolled, from 2006 to 2012. Five were excluded: two due to previous radiotherapy and three due to noncompliance to follow-up. Thus, ten pregnancies in eight patients (age: 24-37 years) completed the study, seven from the same unit in São Paulo.

\section{Baseline characteristics of patients before pregnancy}

Clinical, hormonal, and radiological characteristics of the eight patients (A-H) before each pregnancy $(1,2,3,4,5,6$, $7,8,9,10)$ are summarized in Table 1 . All eight patients had pituitary macroadenomas at diagnosis and had been subjected to transsphenoidal surgery 3-48 months before pregnancy. Patients usually had large tumor residues, mostly in the cavernous sinus, distant $(>0.5 \mathrm{~cm})$ from the optic chiasm. No patient had visual field defects. Duration of previous pharmacological treatment ranged from 2 to 65 months (median: 15 months). Two patients were not receiving drug treatment when pregnancy was diagnosed but both had been treated before: patient A with octreotide-LAR (30 mg/month) for 8 months followed by octreotide-LAR (30 mg/month) and cabergoline (3.5 mg/week) for 2 months until pituitary surgery, 3 months before pregnancy; patient D had stopped drug treatment at first pregnancy (D5), 18 months before second pregnancy (D6).

Seven patients were primigesta and one patient $(F)$ had a fetal loss 2 years before her second pregnancy. Patient $\mathrm{C}$ received fertility treatment (one cycle) of clomiphene acetate and recombinant GNRH elsewhere before her first pregnancy.

No patient had hypertension or overweight before pregnancy. Patient $\mathrm{H}$ developed hypocortisolism after surgery and was kept on oral prednisone $2.5 \mathrm{mg} /$ day. No other pituitary hormone deficits had been diagnosed through clinical and baseline hormone evaluation. Patient $G$ had been diagnosed with prediabetes before and during octreotide treatment and persisted with similar abnormal fasting glycemia during pregnancy.

\section{Clinical outcomes during pregnancy and puerperium}

Most patients did not show any signs or symptoms suggesting worsening of acromegaly activity or tumor growth. However, headache without visual abnormalities developed or worsened in three patients: mild and transient in patients $\mathrm{C}$ and $\mathrm{D}$ (first and third trimester respectively) and moderate/severe throughout pregnancy in patient F. All were successfully treated with oral or i.v. dipirone.

One patient $(\mathrm{H})$, who was kept on oral prednisone, developed preeclampsia: hypertension (20 weeks) followed by proteinuria (30 weeks). Her hypertension was treated with $\alpha$-methyldopa and only resolved completely after delivery. One patient (E) developed gestational diabetes, detected by an oral glucose tolerance 
Table 1 Baseline characteristics of patients before pregnancy.

\begin{tabular}{|c|c|c|c|c|c|c|c|c|}
\hline $\begin{array}{l}\text { Patient/ } \\
\text { pregnancy }\end{array}$ & $\begin{array}{c}\text { Age } \\
\text { (years) }\end{array}$ & $\begin{array}{c}\text { Tumor } \\
\text { residue } \\
(\mathrm{cm})\end{array}$ & $\begin{array}{c}\text { Visual } \\
\text { field } \\
\text { defect }\end{array}$ & $\begin{array}{l}\text { Drug treatment } \\
\text { (months) }\end{array}$ & $\begin{array}{l}\text { Drug treatment } \\
\text { (doses) }\end{array}$ & $\begin{array}{c}\text { Basal GH }{ }^{a} \\
(\mu \mathrm{g} / \mathrm{l})\end{array}$ & $\begin{array}{c}\text { IGF1 }^{\mathrm{b}}(\mathrm{ng} / \mathrm{dl}) / \\
(\mathrm{ULN})^{\mathrm{c}}\end{array}$ & $\begin{array}{c}\Delta \text { IGF1 after } \\
\text { OCT } \pm \text { CAB } \\
(\%)^{d}\end{array}$ \\
\hline $\mathrm{A} / 1$ & 32 & 1.3 & No & $\mathrm{OCT} / \mathrm{CAB}(10)^{\mathrm{e}}$ & No & 1.4 & $300 / 1.0$ & $-21^{f}$ \\
\hline $\mathrm{B} / 2$ & 29 & 1.3 & No & $\mathrm{OCT}(4)$ & $30 \mathrm{mg} / \mathrm{month}$ & 4.3 & $354 / 1.1$ & -51 \\
\hline $\mathrm{C} / 3$ & 35 & 2.0 & No & $\mathrm{OCT/CAB} \mathrm{(8)}$ & $\begin{array}{l}30 \mathrm{mg} / \mathrm{month} / \\
3.5 \mathrm{mg} / \text { week }\end{array}$ & 1.8 & $237 / 0.8$ & -75 \\
\hline $\mathrm{C} / 4$ & 37 & 2.0 & No & OCT (2) & $30 \mathrm{mg} / \mathrm{month}$ & 1.7 & $336 / 1.2$ & $-63^{g}$ \\
\hline $\mathrm{D} / 5$ & 24 & 1.2 & No & OCT/CAB (21) & $\begin{array}{c}30 \mathrm{mg} / \mathrm{month} / \\
1 \mathrm{mg} / \text { week }\end{array}$ & 6.8 & $367 / 1.1$ & -60 \\
\hline $\mathrm{D} / 6$ & 26 & 1.2 & No & $\mathrm{OCT/CAB} \mathrm{(21)}$ & 30 mg/month & 3.7 & $\begin{array}{c}371,247 / 1.2 \\
0.8^{\mathrm{h}}\end{array}$ & $-60^{f}$ \\
\hline $\mathrm{E} / 7$ & 36 & 0.5 & No & $\mathrm{OCT} / \mathrm{CAB}$ (21) & $30 \mathrm{mg} / \mathrm{month}$ & 5.2 & $629 / 2.4$ & -41 \\
\hline $\mathrm{F} / 8$ & 37 & 3.3 & No & OCT (65) & $30 \mathrm{mg} / \mathrm{month}$ & 8.5 & $501 / 1.9$ & -52 \\
\hline $\mathrm{G} / 9$ & 28 & 2.7 & No & OCT (9) & $20 \mathrm{mg} / \mathrm{month}$ & 3.2 & $322 / 1.0$ & -41 \\
\hline $\mathrm{H} / 10$ & 28 & 0.5 & No & OCT (12) & 15 mg/day & $6.3^{\mathrm{i}}, 14.2^{\mathrm{j}}$ & $512 / 1.6$ & +30 \\
\hline
\end{tabular}

test at 26 weeks of pregnancy, and was controlled by diet and resolved in puerperium. The patient $(G)$ who had prediabetes before pregnancy was well controlled with diet and rapid-acting insulin (as blood glucose targets during pregnancy are straight); her diabetes was not considered as gestational diabetes. Two patients (A and C) developed gestational diabetes insipidus (low-density poliuria and polidipsia) during the third trimester of pregnancy and were successfully treated with intranasal desmopressin, with complete recovery after delivery. One patient (D) had uterine bleeding during the second trimester that was resolved with bed rest. One (F) patient had premature uterine contractions from week 17 to week 22; she remained on bed rest and was treated with intravaginal micronized progesterone. Data on clinical follow-up during pregnancy are presented in Table 2 .

None of the seven cases with prepregnancy IGF1 levels below 1.3 ULN developed metabolic complications during pregnancy, whereas two of the three cases with highest prepregnancy IGF1 levels (1.6-2.4 ULN) developed gestational diabetes mellitus (E) and hypertension/ preeclampsia $(\mathrm{H})$. The development of diabetes or

Table 2 Data on clinical and hormonal follow-up of ten pregnancies in eight acromegalic patients.

\begin{tabular}{l} 
Patient/ \\
pregnancy \\
\hline A/1 \\
B/2 \\
C/3 \\
C/4 \\
D/5 \\
D/6 \\
E/7 \\
F/8 \\
G/9 \\
H/10
\end{tabular}

\begin{tabular}{ccc}
$\begin{array}{c}\text { Gestational } \\
\text { diabetes }\end{array}$ & & Hypertension \\
\cline { 1 - 1 } No & & No \\
No & & No \\
No & & No \\
No & & No \\
No & & No \\
No & & No \\
Yes & & No \\
No & & No \\
No & No \\
No & Yes
\end{tabular}

\begin{tabular}{l}
\hline Other events \\
\hline DI \\
No \\
Headache/DI \\
Headache/DI \\
Uterine bleeding \\
Headache \\
No \\
Headache/PUC \\
No \\
Preeclampsia \\
\hline
\end{tabular}

\begin{tabular}{c}
$\begin{array}{c}\text { Visual } \\
\text { changes }\end{array}$ \\
\hline No \\
No \\
No \\
No \\
No \\
No \\
No \\
No \\
No \\
No \\
\hline
\end{tabular}

\begin{tabular}{c}
\hline $\begin{array}{c}\text { Tumor } \\
\text { growth }\end{array}$ \\
\hline No \\
No \\
No \\
No \\
No \\
No \\
No \\
No \\
No \\
No \\
\hline
\end{tabular}

\begin{tabular}{c}
$\begin{array}{c}\text { Newborn } \\
\text { weight }(\mathrm{kg})\end{array}$ \\
\hline 2.850 \\
3.470 \\
3.200 \\
3.550 \\
2.700 \\
2.800 \\
3.490 \\
3.450 \\
3.320 \\
2.180 \\
\hline
\end{tabular}

\begin{tabular}{c}
$\begin{array}{c}\text { Newborn } \\
\text { length }(\mathrm{cm})\end{array}$ \\
\hline 47 \\
47 \\
50 \\
51 \\
46 \\
47 \\
50 \\
51 \\
49 \\
48 \\
\hline
\end{tabular}

Gestational age at delivery (weeks)

DI, diabetes insipidus; PUC, premature uterine contractions.

www.eje-online.org 
hypertension was nonsignificantly associated with prepregnancy IGF1 levels higher than 1.3 ULN $(P=0.06$, Fischer's exact test).

Deliveries were at term in nine cases; one patient (patient $\mathrm{H}$ ) delivered at 35 weeks due to preeclampsia. All newborns were healthy, with normal weight and length for gestational age, and had no malformations at physical examination. Breastfeeding was allowed before pharmacological treatment was resumed. All patients but one breastfed until drug treatment was resumed. In that period, most patients developed, at different time intervals, signs and/or symptoms suggesting increased disease activity such as sweating, headache, edema, and arthralgia.

\section{IGF1 levels}

The IGF1 normal ranges outside pregnancy were assumed as the normal ranges for the Brazilian population (18), and during pregnancy as the ranges obtained from our controls.

As shown in Fig. 1, IGF1 levels were high in all eight patients after surgery (median: 2.8 ULN, range: 1.1-6.2 ULN) and had decreased significantly $(P<0.01)$ before pregnancy $(n=10)$, either during $(n=8)$ or after a temporary suspension of pharmacological treatment $(n=2)$. IGF1 levels before each pregnancy were found in the normal range in four cases, slightly above in three (1.1-1.2 ULN) and high in another three cases ( $>1.2$ ULN). During pregnancy, in spite of pharmacological treatment withdrawn, mean IGF1 levels in patients remained unchanged. However, as the range of IGF1 levels in our healthy control pregnancies was initially lower than the nonpregnant population range, then increased significantly after midgestation, the prevalence of patients with IGF1 levels within each control range also increased significantly from $2 / 10$ ( $\leq 19$ weeks) to $6 / 9$ (20-29 weeks) and $9 / 10$ ( $\geq 30$ weeks) $(P=0.0003)$. The medians and ranges of IGF1 levels in our pregnant controls were, respectively, 0.46 ULN (0.22-0.87 ULN) between 5 and 19 weeks, 0.61 ULN (0.31-1.5 ULN) between 20 and 29 weeks, and 0.85 ULN (0.26-2.0 ULN) between 30 and 39 weeks.

During puerperium, individual IGF1 levels increased in all patients and were significantly higher than those observed before or during pregnancy, and similar to IGF1 levels observed after surgery with no pharmacological treatment. Individual IGF1 levels were already elevated ( $>1.0$ ULN) in 8/9 cases evaluated between 3 and 6 weeks after delivery; in one patient (A), IGF1 levels remained within the normal range for 18 weeks, but eventually

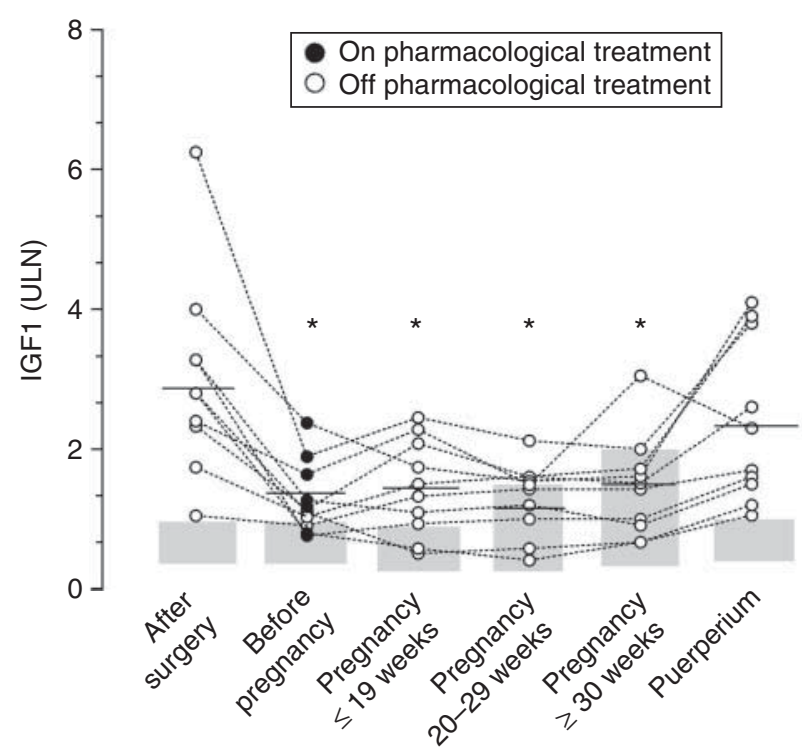

\section{Figure 1}

IGF1 levels (ULN) in ten pregnancies in eight acromegalic patients. Bars (after surgery, before pregnancy, and puerperium) represent the ranges of normal IGF1 values in the Brazilian population or the ranges of IGF1 values obtained in our pregnant controls. Horizontal lines represent mean values from patients. ${ }^{\star} P<0.05$ vs after surgery (repeated measures ANOVA, Bonferroni's post-test for multiple comparisons).

increased at 34 weeks. In another case (D6), the first IGF1 measurement was only performed at 11 weeks.

\section{GH levels}

As shown in Table 3 and Fig. 2A, GH levels markedly increased (mean: $354 \%$; range: $75-1430 \%$ ) after delivery in five cases (A-D5), but showed only minor variations in the remaining five (cases D6-H) (mean: $-17 \%$; range: -33 to $-12 \%)$ as compared with prepregnancy GH levels observed during GH-suppressing treatment. Comparison of prepregnancy IGF1 responses to GH-suppressing treatment between patients with marked increases in GH levels after delivery and those with only minor variations showed greater responses to previous drug treatment in those with larger post-pregnancy GH increase ( -62 vs $-26 \%$, respectively, $P=0.057)$, but no significant difference was found in duration of octreotide/cabergoline treatment between them $(P=0.15)$.

During pregnancy, however, GH levels were assessed by the interference-free $\mathrm{GH}$ assay only in those five patients who showed minor variations in GH levels after 
Table 3 Data on $\mathrm{GH}$ concentrations before and after pregnancy.

\begin{tabular}{|c|c|c|c|c|}
\hline $\begin{array}{l}\text { Patient/ } \\
\text { pregnancy }\end{array}$ & $\begin{array}{c}\text { GH } \\
\text { measured } \\
\text { during } \\
\text { pregnancy }\end{array}$ & $\begin{array}{c}\text { Prepregnancy } \\
\text { GH }(\mu \mathrm{g} / \mathrm{l})\end{array}$ & $\begin{array}{c}\text { Puerperal } \\
\text { GH }(\mu \mathrm{g} / \mathrm{l})\end{array}$ & $\begin{array}{l}\Delta \mathbf{G H} \\
(\%)^{\mathrm{a}}\end{array}$ \\
\hline $\mathrm{A} / 1$ & No & 1.4 & 3.0 & 114 \\
\hline $\mathrm{B} / 2$ & No & 4.3 & 65.7 & 1430 \\
\hline$C / 3$ & No & 1.8 & 3.2 & 78 \\
\hline$C / 4$ & No & 1.7 & 3.1 & 76 \\
\hline $\mathrm{D} / 5$ & No & 6.8 & 11.9 & 75 \\
\hline D/6 & Yes & 3.7 & 3.1 & -16 \\
\hline $\mathrm{E} / 7$ & Yes & 5.2 & 3.5 & -33 \\
\hline$F / 8$ & Yes & 8.5 & 8.2 & -4 \\
\hline $\mathrm{G} / 9$ & Yes & 3.2 & 2.8 & -12 \\
\hline $\mathrm{H} / 10$ & Yes & 6.3 & 4.9 & -21 \\
\hline
\end{tabular}

${ }^{a}(($ Prepregnancy $\mathrm{GH}$, on octreotide \pm cabergoline treatment-postsurgery $\mathrm{GH}) /$ postsurgery $\mathrm{GH}$ concentrations) $\times 100$.

delivery and less important prepregnancy IGF1 responses to drug treatment. In those patients, no significant changes $(P=0.67$, ANOVA) in $\mathrm{GH}$ levels were observed throughout pregnancy, whereas a significant decline in $\mathrm{GH}$ was observed in normal pregnant controls using that same assay $(P=0.0002$, post test for linear trend). One patient (D6), who showed a marked but transient increase in GH levels in midgestation, had received a single dose of octreotide-LAR (30 mg) at 5 weeks of gestation, just before pregnancy was diagnosed. She had been off pharmacological treatment for the last 18 months and had previously responded to octreotide and cabergoline treatment before her first gestation (D5) (Fig. 2B).

\section{Tumor growth}

As shown in Fig. 3, tumor remnants were predominantly extrasellar, in the cavernous sinus, and distant $(\geq 0.5 \mathrm{~cm})$ from the optic chiasm in all but one case that had an intrassellar tumor remnant $(\mathrm{H})$. No changes in tumor size or tumor signal intensity suggesting bleeding were detected after pregnancy.

\section{Discussion}

This study was designed to improve our understanding on the main clinical, biochemical, maternal-fetal, and tumorrelated outcomes in acromegalic patients during pregnancy and puerperium. Our patients comprise a representative cohort of the usual acromegalic patient with variable disease control under pharmacological treatment after a noncurative surgery for a pituitary macroadenoma.

\section{Clinical activity of acromegaly during pregnancy and puerperium}

Although no clear signs or symptoms of disease activity developed after the treatment was discontinued, headache without visual changes was a common complaint during pregnancy. It is important that headache could not be attributed to tumor enlargement, as shown by the unchanged size of the tumors after delivery, or to IGF1 levels, which remained stable throughout gestation. Increasing GH levels, however, may be related to the development of headache in some patients following treatment withdrawal. On the other hand, most patients had been discontinued from octreotide, a somatostatin analog that can have a dramatic analgesic effect on acromegaly-associated headache; such effect is not exclusively associated with GH suppression and could result from the inhibition of an unknown pro-nociceptive peptide $(20,21)$. In effect, all patients that reported headaches during gestation had also reported headache before pharmacological treatment and improved markedly after octreotide, irrespective of hormonal control. Accordingly, octreotide withdrawal was likely a major contributor for the recurrence of headache in those patients.

The prevalence of hypertension and diabetes during pregnancy in our patients was unremarkable in relation to the expected prevalences in the Brazilian population (1/10 and $1 / 10$ vs $7.4 / 100$ and $7.6 / 100$ respectively) $(22,23)$. These data are in accordance with the results of two recent multicentric retrospective studies showing no increase in the overall prevalence of diabetes and hypertension during pregnancy in acromegaly $(2,7)$. Nevertheless, even
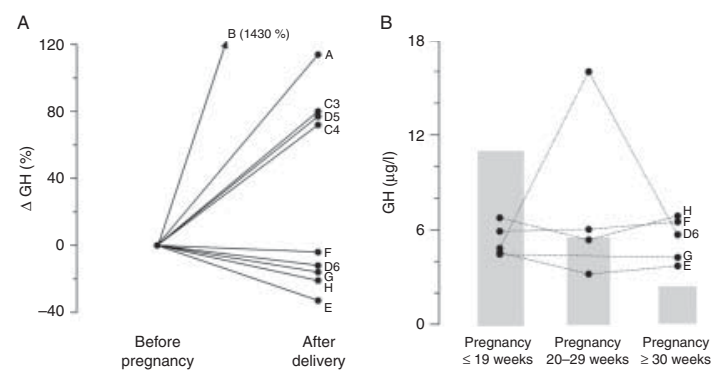

Figure 2

Individual percent variations in serum $\mathrm{GH}$ levels after delivery in ten acromegalic pregnancies (A); GH levels measured by an interference-free $\mathrm{GH}$ assay during pregnancy in five acromegalic patients after pharmacological treatment withdrawal. Gray bars represent the ranges (minimum-maximum) of serum GH levels in 64 control pregnancies (B). 

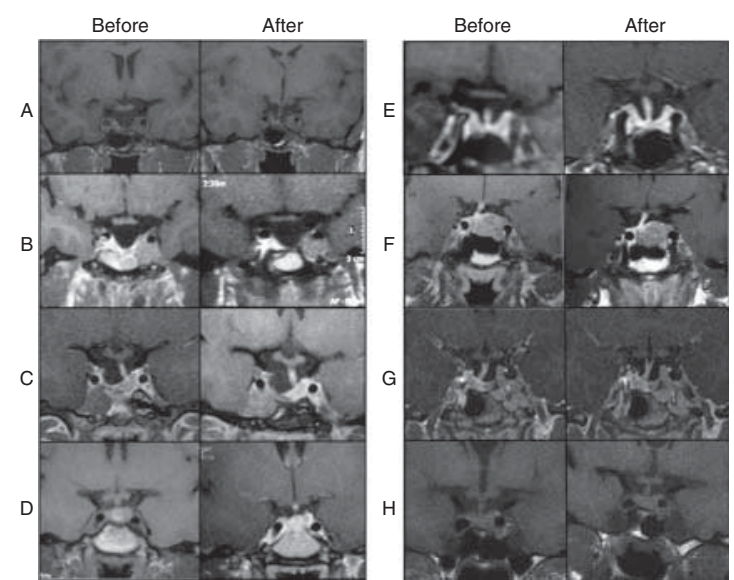

\section{Figure 3}

Sellar magnetic resonance images before (left, in each column) and after (right, in each column) pregnancy in ten pregnancies in eight acromegalic patients. Images $C$ and D were obtained before the largest of those two studies did not have statistical power to detect significance in a twofold increase in the prevalence of both conditions observed in patients with uncontrolled prepregnancy GH/IGF1 levels (2). In our study, it is noteworthy that such complications developed in two of the three patients with highest prepregnancy IGF1 levels and in none of the remaining ones, all of which had controlled or nearly controlled prepregnancy IGF1 levels ( $<1.3 \mathrm{ULN})$, but the number of complications was too small to allow for statistical significance. Thus, it is not unlikely that uncontrolled acromegaly before pregnancy may pose some additional risk for the development of diabetes and hypertension during pregnancy.

The development of gestational diabetes insipidus during late gestation, observed in two patients, was likely the result from the combined effects of a reduced vasopressin reserve due to previous pituitary surgery and the physiological increase in placental vasopressinase activity in late pregnancy. Recurrence of signs and/or symptoms of acromegaly activity occurred in puerperium and were paralleled by increased IGF1 levels. The overall beneficial effect of pregnancy on both clinical and hormonal activity of acromegaly is transient and usually disappears within a few weeks after delivery.

\section{Obstetrical/fetal outcomes and lactation}

The relatively uneventful course of pregnancy and delivery and the healthy newborns in our series suggest that withdrawal of octreotide and cabergoline treatment during pregnancy is also safe for obstetrical and fetal outcomes. Octreotide crosses the placental barrier, is present in maternal milk, and has been shown to decrease fetal size (14). Cabergoline, on the other hand, is considered safe during pregnancy (15), but impairs lactation. Although our patients usually achieved control of IGF1 levels during pregnancy, both maternal GH and IGF1 levels do not cross the placental level (24). During puerperium, lactation was feasible and most patients were successful in breastfeeding before pharmacological treatment was restarted.

\section{GH levels during pregnancy and puerperium}

The development and application of our noncrossreactive immunometric GH assay to assess GH levels throughout pregnancy confirmed the autonomy of tumoral GH secretion during gestation, which had been clearly demonstrated in only two patients using a noncrossreactive GH RIA (25). Since prepregnancy and puerperal basal GH levels were measured by other assays in our patients, comparisons of those values with measurements obtained during pregnancy could not be made. However, comparisons between individual pre- and post-pregnancy GH levels by the same assay showed that patients with no elevations in GH concentrations after delivery had usually shown the lowest IGF1 responses during prepregnancy treatment with GH-suppressing drugs. In addition, their GH levels did not change during pregnancy as shown by the interference-free assay. On the other hand, patients with markedly increased GH levels after delivery had shown greater IGF1 suppression during prepregnancy octreotide and/or cabergoline treatment. Unfortunately, as in those patients GH levels had not been assessed during pregnancy by the interference-free assay, the timing of the GH rise could not be determined. However, it is likely that GH levels in those patients rebounded during pregnancy as the effect of the drugs disappeared, but the time course could be highly variable since the effects of octreotide may last long after its suspension (26). At any rate, these data indicate that pregnancy does not further stimulate the secretory activity of somatotrophic adenomas.

\section{IGF1 levels during pregnancy and puerperium}

In spite of pharmacological treatment withdrawal, IGF1 levels in our acromegalic patients remained relatively unchanged during pregnancy and comparable with IGF1 levels obtained during pharmacological treatment. 
Thus, both pharmacological treatment before pregnancy and pregnancy without pharmacological treatment were similarly effective in suppressing IGF1 concentrations. The powerful blocking effect of pregnancy on GH-induced IGF1 generation was remarkably illustrated in one of our patients who showed a striking rebound of GH secretion during midgestation with no change in serum IGF1 concentrations.

The effect of pregnancy on IGF1 levels is likely to reflect the blockade of IGF1 generation by the strikingly high estrogen levels of pregnancy (27), which can reach by term $\sim 200$ times that of the nonpregnant state (28). Oral estrogen has been used to treat acromegaly in the past, with clinical improvement, and decreased IGF1 levels in acromegalic patients (29). Estrogen has been shown to inhibit GH signaling by suppressing GH-dependent activation of the JAK/STAT pathway, in a dose-dependent manner, an effect mediated by SOCS2. Although the blocking effect of estrogen on IGF1 generation is partially overcome in mid/late normal pregnancy, when pGH concentrations increase, pituitary GH concentrations decrease, and IGF1 levels rise $(30,31)$, a similar IGF1 elevation was not observed in our patients. Assuming that both estrogen and pGH secretion profiles during acromegalic pregnancy are similar to normal pregnancy, one would expect further increases in IGF1 levels due to the growing contribution of $\mathrm{pGH}$ after midgestation.

IGF1 levels in patients were already increased during the first trimester (as tumoral GH secretion is not progressively suppressed as in controls), but remained stable thereafter. The apparent paradox of increasing $\mathrm{pGH}$ with no further increases in IGF1 levels after midgestation in our acromegalic patients may reflect that the tumoral GH levels of our patients (range 3.7-8.4 $\mu \mathrm{g} / \mathrm{l}, \geq 29$ weeks) could not be significant to add somatogenic effect to much higher pGH concentrations (range 2.1-69.8 $\mu \mathrm{g} / \mathrm{l}$, third trimester) (32), in a situation of blunted maximal response of hepatic IGF1 generation.

After delivery, when the influences of both pGH and estrogen had completely ceased, IGF1 levels increased markedly. Thus, estrogen-induced GH resistance is likely to be a major factor keeping IGF1 levels stable in spite of increasing $\mathrm{pGH}$ and unrestrained tumor $\mathrm{GH}$ secretion during pregnancy.

On the other hand, as IGF1 concentrations increased during pregnancy in our controls, the prevalence of patients with IGF1 levels within the control range for gestational age increased significantly throughout pregnancy. In effect, the prevalence of patients with controlled IGF1 levels increased more than twofold from pre-pregnancy to late pregnancy. In puerperium, IGF1 levels returned to pre-treatment levels in a few weeks after delivery and patients had to resume pharmacological treatment to control clinical and hormonal disease activity. These data clearly indicate that maintaining drug therapy in acromegalic patients during pregnancy is usually unnecessary to keep control of disease activity.

\section{Tumor growth}

As no tumor enlargement occurred during pregnancy in our series of ten pregnancies in eight patients without previous pituitary radiotherapy, pregnancy is not expected to stimulate growth of most somatotrophic adenomas. In a large retrospective series, only three of 27 cases (11\%) (2) exhibited radiological evidence of tumor growth during pregnancy. However, as the pituitary gland enlarges during gestation due to hyperplasia of lactotrophic cells, pregnant patients with macroadenomas may also develop visual symptoms as a result of the pituitary enlargement in a restricted sellar space (33). No visual field defects developed in our patients, but they had all been previously operated and all tumor remnants were distant from the optic chiasm. Tumor enlargement after octreotide withdrawal (34), tumor apoplexy $(35,36)$, aggressive tumors (37), and untreated acromegaly $(38,39,40,41)$ may determine a less favorable visual outcome during pregnancy.

\section{Conclusions}

In conclusion, pregnancy in acromegaly usually improves disease control and does not stimulate tumor growth, even after drug treatment withdrawal, and newborns are usually healthy. Drug treatment for acromegaly can be discontinued during pregnancy in most cases. Pregnancy following effective control of IGF1 by pharmacological treatment does not pose additional risks for diabetes mellitus and/or hypertension during pregnancy, but patients with uncontrolled disease before pregnancy may be at a higher risk for those complications.

\section{Declaration of interest}

The authors declare that there is no conflict of interest that could be perceived as prejudicing the impartiality of the research reported.

\section{Funding}

This research did not receive any specific grant from any funding agency in the public, commercial or not-for-profit sector. 


\section{Acknowledgements}

The authors are grateful to Leda Hitomi Obara, Teresinha Tachibana, Teresa Kasamatsu, Aparecida Filomena Machado, and Ivonete Alves Carvalhaes for their kind and valuable technical assistance in the GH assays and in blood collection (I.A.C)

\section{References}

1 Grynberg M, Salenave S, Young J \& Chanson P. Female gonadal function before and after treatment of acromegaly. Journal of Clinical Endocrinology and Metabolism 201095 4518-4525. (doi:10.1210/jc.2009-2815)

2 Caron P, Broussaud S, Bertherat J, Borson-Chazot F, Brue T, CortetRudelli C \& Chanson P. Acromegaly and pregnancy: a retrospective multicenter study of 59 pregnancies in 46 women. Journal of Clinical Endocrinology and Metabolism 201095 4680-4687. (doi:10.1210/jc. 2009-2331)

3 Molitch ME. Pregnancy and the hyperprolactinemic woman. New England Journal of Medicine 1985312 1364-1370. (doi:10.1056/ NEJM198505233122106)

4 Goluboff LG \& Ezrin C. Effect of pregnancy on the somatotroph and the prolactin cell of the human adenohypophysis. Journal of Clinical Endocrinology and Metabolism 196929 1533-1538. (doi:10.1210/ jcem-29-12-1533)

5 Cheng V, Faiman C, Kennedy L, Khoury F, Hatipoglu B, Weil R \& Hamrahian A. Pregnancy and acromegaly: a review. Pituitary 201215 59-63. (doi:10.1007/s11102-011-0330-3)

6 Shimatsu A, Usui T, Tagami T, Kuzuya H \& Takahashi JA. Suppressed levels of growth hormone and insulin-like growth factor-1 during successful pregnancy in persistent acromegaly. Endocrine Journal 2010 57 551-553. (doi:10.1507/endocrj.K10E-069)

7 Lau SL, McGrath S, Evain-Brion D \& Smith R. Clinical and biochemical improvement in acromegaly during pregnancy. Journal of Endocrinological Investigation 200831 255-261.

8 Cheng S, Grasso L, Martinez-Orozco JA, Al-Agha R, Pivonello R, Colao A $\&$ Ezzat S. Pregnancy in acromegaly: experience from two referral centers and systematic review of the literature. Clinical Endocrinology 201276 264-271. (doi:10.1111/j.1365-2265.2011.04180.x)

9 Abelove WA, Rupp JJ \& Paschkis KE. Acromegaly and pregnancy. Journal of Clinical Endocrinology and Metabolism 195414 32-44. (doi:10.1210/jcem-14-1-32)

10 Obuobie K, Mullik V, Jones C, John R, Rees AE, Davies JS, Scanlon MF \& Lazarus JH. McCune-Albright syndrome: growth hormone dynamics in pregnancy. Journal of Clinical Endocrinology and Metabolism 200186 2456-2458. (doi:10.1210/jc.86.6.2456)

11 Dias ML, Vieira JG \& Abucham J. Detecting and solving the interference of pregnancy serum, in a GH immunometric assay. Growth Hormone \& IGF Research 201323 13-18. (doi:10.1016/j.ghir.2012.11.001)

12 Frankenne F, Closset J, Gomez F, Scippo ML, Smal J \& Hennen G. The physiology of growth hormones (GHs) in pregnant women and partial characterization of the placental GH variant. Journal of Clinical Endocrinology and Metabolism 198866 1171-1180. (doi:10.1210/ jcem-66-6-1171)

13 Melmed S, Casanueva FF, Klibanski A, Bronstein MD, Chanson P, Lamberts SW, Strasburger CJ, Wass JA \& Giustina A. A consensus on the diagnosis and treatment of acromegaly complications. Pituitary 2012 16 294-302. (doi:10.1007/s11102-012-0420-x)

14 Maffei P, Tamagno G, Nardelli GB, Videau C, Menegazzo C, Milan G, Calcagno A, Martini C, Vettor R, Epelbaum J et al. Effects of octreotide exposure during pregnancy in acromegaly. Clinical Endocrinology 2010 72 668-677. (doi:10.1111/j.1365-2265.2009.03706.x)

15 Colao A, Abs R, Barcena DG, Chanson P, Paulus W \& Kleinberg DL. Pregnancy outcomes following cabergoline treatment: extended results from a 12-year observational study. Clinical Endocrinology 200868 66-71. (doi:10.1111/j.1365-2265.2007.03000.x)
16 Qureshi A, Kalu E, Ramanathan G, Bano G, Croucher C \& Panahloo A. IVF ICSI in a woman with active acromegaly: successful outcome following treatment with pegvisomant. Journal of Assisted Reproduction and Genetics 200623 439-442. (doi:10.1007/s10815-006-9077-6)

17 Brian SR, Bidlingmaier M, Wajnrajch MP, Weinzimer SA \& Inzucchi SE. Treatment of acromegaly with pegvisomant during pregnancy: maternal and fetal effects. Journal of Clinical Endocrinology and Metabolism 200792 3374-3377. (doi:10.1210/jc.2007-0997)

18 Rosario PW. Normal values of serum IGF-1 in adults: results from a Brazilian population. Arquivos Brasileiros de Endocrinologia e Metabologia 201054 477-481. (doi:10.1590/S0004-27302010000500008)

19 Vieira JG, Lombardi MT \& Nishida SK. Monoclonal antibody-based immunoenzymometric assay for serum human growth hormone. Brazilian Journal of Medical and Biological Research 199023 293-296.

20 Levy MJ, Bejon P, Barakat M, Goadsby PJ \& Meeran K. Acromegaly: a unique human headache model. Headache 200343 794-797. (doi:10.1046/j.1526-4610.2003.03139.x)

21 Matharu MS, Levy MJ, Meeran K \& Goadsby PJ. Subcutaneous octreotide in cluster headache: randomized placebo-controlled double-blind crossover study. Annals of Neurology 200456 488-494. (doi:10.1002/ana.20210)

22 Oliveira CA, Lins CP, Sá RAM, Netto HC, Bornia RG, Silva NR \& Junior JA. Hypertensive syndromes during pregnancy and perinatal outcomes. Revista Brasileira de Saúde Materno Infantil 2006 93-98. (doi:10.1590/S1519-38292006000100011)

23 Detsch JC, Almeida AC, Bortolini LG, Nascimento DJ, Oliveira Junior FC \& Rea RR. Markers of diagnosis and treatment in 924 pregnancies with gestational diabetes mellitus. Arquivos Brasileiros de Endocrinologia e Metabologia 201155 389-398. (doi:10.1590/S000427302011000600005)

24 Verhaeghe J. Does the physiological acromegaly of pregnancy benefit the fetus? Gynecologic and Obstetric Investigation 200866 217-226. (doi:10.1159/000147167)

25 Beckers A, Stevenaert A, Foidart JM, Hennen G \& Frankenne F. Placental and pituitary growth hormone secretion during pregnancy in acromegalic women. Journal of Clinical Endocrinology and Metabolism 199071 725-731. (doi:10.1210/jcem-71-3-725)

26 Ramirez C, Vargas G, Gonzalez B, Grossman A, Rabago J, Sosa E, Espinosa-de-Los-Monteros AL \& Mercado M. Discontinuation of octreotide LAR after long term, successful treatment of patients with acromegaly: is it worth trying? European Journal of Endocrinology 2012 166 21-26. (doi:10.1530/EJE-11-0738)

27 Feldt-Rasmussen U \& Mathiesen ER. Endocrine disorders in pregnancy: physiological and hormonal aspects of pregnancy. Best Practice \& Research. Clinical Endocrinology \& Metabolism 201125 875-884. (doi:10.1016/j.beem.2011.07.004)

28 Leung KC, Johannsson G, Leong GM \& Ho KK. Estrogen regulation of growth hormone action. Endocrine Reviews 200425 693-721. (doi:10.1210/er.2003-0035)

29 Shimon I \& Barkan A. Estrogen treatment for acromegaly. Pituitary 2012 15 601-607. (doi:10.1007/s11102-012-0426-4)

30 Fuglsang J \& Ovesen P. Aspects of placental growth hormone physiology. Growth Hormone \& IGF Research 200616 67-85. (doi:10.1016/j.ghir.2006.03.010)

31 Chellakooty M, Vangsgaard K, Larsen T, Scheike T, Falck-Larsen J, Legarth J, Andersson AM, Main KM, Skakkebaek NE \& Juul A. A longitudinal study of intrauterine growth and the placental growth hormone (GH)-insulin-like growth factor I axis in maternal circulation: association between placental GH and fetal growth. Journal of Clinical Endocrinology and Metabolism 200489 384-391. (doi:10.1210/jc.2003030282)

32 Wu Z, Bidlingmaier M, Friess SC, Kirk SE, Buchinger P, Schiessl B \& Strasburger C. A new nonisotopic, highly sensitive assay for the measurement of human placental growth hormone: development and clinical implications. Journal of Clinical Endocrinology and Metabolism 200388 804-811. (doi:10.1210/jc.2002-020787) 
33 Kupersmith MJ, Rosenberg C \& Kleinberg D. Visual loss in pregnant women with pituitary adenomas. Annals of Internal Medicine 1994121 473-477. (doi:10.7326/0003-4819-121-7-199410010-00001)

34 Cozzi R, Attanasio R \& Barausse M. Pregnancy in acromegaly: a onecenter experience. European Journal of Endocrinology 2006155 279-284. (doi:10.1530/eje.1.02215)

35 Atmaca A, Dagdelen S \& Erbas T. Follow-up of pregnancy in acromegalic women: different presentations and outcomes. Experimental and Clinical Endocrinology \& Diabetes 2006114 135-139. (doi:10.1055/s-2005-873004)

36 Lunardi P, Rizzo A, Missori P \& Fraioli B. Pituitary apoplexy in an acromegalic woman operated on during pregnancy by transphenoidal approach. International Journal of Gynaecology and Obstetrics 199134 71-74. (doi:10.1016/0020-7292(91)90542-D)

37 Kasuki L, Neto LV, Takiya CM \& Gadelha MR. Growth of an aggressive tumor during pregnancy in an acromegalic patient. Endocrine Journal 201259 313-319. (doi:10.1507/endocrj.EJ11-0306)
38 Guven S, Durukan T, Berker M, Basaran A, Saygan-Karamursel B \& Palaoglu S. A case of acromegaly in pregnancy: concomitant transsphenoidaladenomectomy and cesarean section. Journal of Maternal-Fetal \& Neonatal Medicine 200619 69-71. (doi:10.1080/ 14767050500434021)

39 Hisano M, Sakata M, Watanabe N, Kitagawa M, Murashima A \& Yamaguchi K. An acromegalic woman first diagnosed in pregnancy. Archives of Gynecology and Obstetrics 2006274 171-173. (doi:10.1007/ s00404-005-0114-y)

40 Takeuchi K, Funakoshi T, Oomori S \& Maruo T. Successful pregnancy in an acromegalic women treated with octreotide. Obstetrics and Gynecology 199993 848. (doi:10.1016/S00297844(98)00461-X)

41 Yap AS, Clouston WM, Mortimer RH \& Drake RF. Acromegaly first diagnosed in pregnancy: the role of bromocriptine therapy. American Journal of Obstetrics and Gynecology 1990163 477-478. (doi:10.1016/ 0002-9378(90)91178-F)

Received 31 May 2013

Revised version received 13 November 2013

Accepted 18 November 2013 